\title{
Psychosocial Issues Associated with Breast Cancer-Related Lymphedema: a Literature Review
}

\author{
L. H. Eaton ${ }^{1} \cdot$ N. Narkthong ${ }^{2} \cdot$ J. M. Hulett ${ }^{2}$ \\ Published online: 25 August 2020 \\ (C) Springer Science+Business Media, LLC, part of Springer Nature 2020
}

\begin{abstract}
Purpose of Review Breast cancer-related lymphedema (BCRL) is a chronic disease affecting breast cancer survivors. The purpose of this article is to update the scientific literature regarding psychosocial issues associated with BCRL.

Recent Findings Reports describe economic burdens, social support, sexuality, BCRL patient-education needs, and interventions to reduce BCRL symptoms and improve QOL among women with breast cancer. The psychosocial impact of BCRL may differ between younger and older women which has implications for age-related interventions to reduce the adverse psychosocial experiences of women with BCRL. We did not locate studies reporting the psychosocial impact of BCRL on male breast cancer survivors.

Summary More psychosocial-based interventions are needed that target the concerns of those with BCRL, including age-related needs, sexual concerns, body image, and social support. Future research is indicated to study the psychosocial impact of BCRL among men. Researchers may consider how pandemic-driven health care policies affect the psychosocial needs of those with BCRL.
\end{abstract}

Keywords Breast Cancer $\cdot$ Literature review $\cdot$ Lymphedema $\cdot$ Psychosocial $\cdot$ Quality of life $\cdot$ Treatment

\section{Introduction}

Breast cancer is the most common cancer among females in the USA [1]. Breast cancer-related lymphedema (BCRL), the accumulation of protein-rich fluid in the tissues due to insufficient lymph drainage, may occur after lymph node disruption during breast cancer treatment [2]. These treatments may include surgical removal of axillary lymph nodes and

This article is part of the Topical Collection on Lymphedema Incidence, Prevention and Treatment

J. M. Hulett

hulettjm@missouri.edu

L. H. Eaton

lineaton@uw.edu

N. Narkthong

Nnqc4@mail.missouri.edu

1 School of Nursing \& Health Studies, University of Washington Bothell, Box 358531, Bothell, WA 98011, USA

2 Sinclair School of Nursing, University of Missouri, 115 Business Loop 70W, Mizzou North, Room 408C,

Columbia, MO 65211-6000, USA radiation therapy to the breast and axilla and, thus, may result in swelling of the upper extremity that generally worsens over time [3]. Previous research indicates that individuals who received axillary lymph node dissection have a higher incidence of BCRL (33.3\%) than individuals who received sentinel lymph node biopsy $(3.4 \%)(p<.001)$ at 5 years posttreatment for breast cancer [4०•]. Armer and Stewart [5] reported that the lifetime risk of developing BCRL ranges from 43 to $94 \%$ by 5 years post-treatment, depending on the BCRL criterion measure. Bell et al. (2013) reported that $20 \%$ of women treated for breast cancer $(N=1588)$ were diagnosed with BCRL by 2 years post-diagnosis [6].

Physical symptoms, such as pain, infection, and impaired arm mobility, are known to occur with BCRL and adversely affect physical functioning and psychological health, subsequently resulting in decreased quality of life (QOL) [7, 8]. The physical and psychosocial aspects of BCRL have been a focus of many research studies. Interventions for BCRL management have been evaluated for efficacy and effect on QOL, while specific factors of psychological health (e.g., anxiety, depression, body image) have been reported. In a systematic review of 23 articles published from January 2004 to December 2011, Fu et al. [8] reported on the negative 
psychosocial impact of BCRL on breast cancer survivors and identified a need for BCRL-specific instruments to describe the psychosocial impact of BCRL [8]. The purpose of this paper is to conduct a literature review, building on the systematic review by $\mathrm{Fu}$ and colleagues [8] and summarizing the scientific literature from 2010 to February 2020 on the psychosocial issues among those with BCRL. Our review is guided by the question: What are the psychosocial issues associated with BCRL?

\section{Literature Search}

We searched the following databases from January 1, 2010, to February 26, 2020: PubMed, MEDLINE, CINAHL, the Cochrane Database of Systematic Reviews, Google Scholar, Scopus, and Web of Science. We excluded gray literature, case reports, letters to the editor, commentaries, articles in which full articles were not available, and non-breast cancerrelated articles. Search filters were peer-reviewed, full-article available in English, and studies of adults over 19 years old. Initial key search terms included lymphedema, lymphodema, lymphoedema, elephantiasis, swelling, oedema. Search terms were expanded to include (1) psychosocial impact, psychosocial consequences, psychosocial concerns; (2) quality of life, well-being, emotional well-being, emotional distress, spiritual well-being, spiritual distress; (3) body image, appearance, sexuality, intimacy; (4) social barriers, social support, social isolation, public insensitivity, social abandonment, work environment, marginalization, return to work, stigma, congregational support; (5) psychological distress, psychological impact, depression, anxiety, fear, mood/mood disturbance; (6) financial burden, economic burden, hardship, lack of health care, lack of health insurance; and (7) gender, male breast cancer.

The following is a synthesis of the psychosocial research conducted during the past 10 years focused on psychosocial aspects of diagnosis with BCRL.

\section{Psychosocial Issues in BCRL}

\section{Physical Health Factors}

Pain and Upper Extremity Disability Our review found that upper extremity pain and impaired limb function remain two primary issues affecting QOL among individuals with BCRL. Researchers report that individuals with BCRL have more upper extremity impairment and limitations than those without BCRL [9•, 10]. Breast cancer survivors with upper extremity pain, impaired limb movement, and pain of the operated breast experience poorer QOL and greater psychological distress $[11,12]$. In contrast, other researchers have reported that impaired upper extremity function was associated with physical and functional well-being, but not emotional or social well-being [13]. Another group of researchers found that BCRL was not associated with QOL among women who had received sentinel lymph node biopsy [10]. Interestingly, it has been reported that the negative impact of BCRL on QOL may also negate the positive QOL impact of having had reconstructive surgery after mastectomy [14]. Finally, a pilot study by Jeong et al. [15] reported that shoulder pain after BCRL diagnosis $(N=39)$ is associated with reduced QOL $(p<.05)$. Furthermore, shoulder pain may be indicative of shoulder pathology (e.g., rotator cuff injury) rather than BCRL. Jeong's [15] study underscores that not all shoulder pain after BCRL is related to BCRL; and, determining the cause of shoulder pain in women diagnosed with BCRL is necessary to treat shoulder pathology that is curable. Indeed, over half of the study's participants also reported adhesive capsulitis (i.e., "frozen shoulder" symptoms) [15].

\section{Psychological Health Factors}

Psychological Distress Researchers report that BCRL is associated with poorer psychosocial well-being and poorer QOL due to symptoms related to anxiety, depression, emotional distress, fatigue, self-care, relationship issues, impaired mobility, or ability to participate in social activities [6, 16-19]. Common symptom clusters studied in relationship with BCRL include pain, fatigue, and distress [20•]. In a study of breast cancer survivors $(N=2431)$, nearly one-third $(28.5 \%)$ $(n=692)$ reported BCRL, and among those reporting BCRL, nearly half $(48.9 \%)(n=1189)$ reported moderate to extreme distress as a result of their BCRL [21]. Furthermore, those with lymphedema-related distress were more likely to report poor physical and mental health than breast cancer survivors without lymphedema [21]. In one study, researchers observed that physical, emotional, and functional well-being improved by 1 year after surgical treatment of breast cancer $(N=196)$, while social well-being decreased [22]. Additionally, at 1 year post-surgery, the incidence of lymphedema was $26.1 \%$ among those who underwent mastectomy $(n=99)$, and $47.6 \%$ among those who underwent breast conserving surgery $(n=97)$ [22].

Body Image Disturbance Several studies reported similar findings regarding the impact of BCRL on body image. Researchers reported that women with BCRL $(N=166)$ experienced depression, anxiety, and stress while perceptions regarding the effectiveness of controlling and treating BCRL further influenced these symptoms [23]. Furthermore, older women reported more body image disturbance and distress [23]. Another group reported that among women with BCRL $(N=181)$, body image, self-esteem, and BCRL presence were factors influencing depression [24]. Similar findings were noted by another study in which women with BCRL 
$(N=97)$ reported frustration, depression, greater irritability, and body image disturbance [25]. Finally, researchers report that pain intensity and body image are factors related to depression among those with BCRL $(N=54)[26]$.

Sexuality Sexuality is significantly influenced and impaired by BCRL. Compared with pretreatment levels, considerably more women with BCRL $(N=191)$ reported moderate or severe difficulties with sexual interest and sexual activity at 3, 6, and 12 months after surgery [27]. Moreover, interest in sexual activity improved gradually during the year after surgery; however, moderate or severe problems with sexual interest and activity persisted over time due to upper limb dysfunction secondary to BCRL [27]. Compared with women without BCRL $(n=109)$, women with BCRL $(n=243)$ reported struggles with compression garments and sexual intimacy, negative feelings involving the breast and arm, and feelings of decreased sexual desire [28]. A study examining the impact of BCRL on sexual functioning reported that a supportive partner was essential in assisting women to overcome sexual issues caused by severe swelling and/or body image concerns $(N=17)[29]$.

Social Support The social sequela of BCRL is well-described in a review by Tsuchiya [30] which notes the social impact of BCRL encompasses employment concerns, social confidence, sexuality, relationships, and lack of privacy due to the visibility of BCRL and compression garments associated with BCRL. Researchers report that women with BCRL $(n=26)$ have poorer social functioning than women without BCRL $(n=119)$ [31]; and social well-being appears to worsen over time [22]. The social construction of gender roles occurring with family and community living may make self-care particularly challenging for women at risk for BCRL as they often struggle with time management and prioritizing self-care over the care of others as well as making a commitment to self-care [32]. Furthermore, the self-care experiences of women with breast cancer are influenced by the complexities and demands of familial and work-related responsibilities [33]. Researchers examining influences on whether breast cancer survivors disclose BCRL to their social networks observed that perceived responsibility of social roles within the family and unsupportive reactions to breast cancer from others are the main influences on whether individuals disclose BCRL. Moreover, support programs for breast cancer survivors who feel unable to disclose BCRL symptoms to family members should be considered in survivorship care planning [34].

Quality of Life A number of studies have examined the differences in QOL between breast cancer survivors with and without BCRL. A study exploring the perceptions of QOL (Functional Assessment of Cancer Therapy (FACT B+4)) and the prevalence of BCRL using subjective and objective measures of lymphedema (i.e., Morbidity Screening Tool (MST); Lymphedema and Breast Cancer Questionnaire (LBCQ); and perometry) found that differences in perceptions of QOL were significant for self-reported measures $(N=617)$ $(p<.05)$; however, objective measurement (perometry) was not related to QOL [35]. Similar findings were observed by two other research groups which reported that subjective perceptions about BCRL influence QOL more than objective evidence of BCRL [36, 37]. Additionally, another group found associations between older age and poorer QOL among those with BCRL $(N=201)$ [9•]. In contrast, three studies reported the presence of BCRL was not associated with QOL [38-40]. Differences between study designs, including sample characteristics (e.g., age of participants and diseasespecific exclusion criteria) [39] and instruments (SF-12, SF36, and FACT-B) used to assess QOL, may account for these findings.

Economic Burden BCRL can significantly impact employment and limit return-to-work [41, 42•]. The cascading nature of the economic burden on long-term savings and work opportunities, and insufficiency of insurance to cover lymphedema needs drove financial differences [43]. Women without BCRL were more likely to return-to-work [44]. Medical chart data indicate that BCRL is associated with a lower return-towork rate and longer delay of return-to-work. Moreover, physical, psychological, and organizational constraints influence the return-to-work rate [45]. Symptoms such as pain, fatigue, BCRL, reduced range of motion and weakness in the upper limbs, and physical limitations lead to the difficulty or impossibility of performing work tasks [46, 47]. However, reduced work engagement and ability, employment status, and work performance are associated with a combination of individual factors, work environment, culture, and resources [48•]. Thus, occupational physicians should assess BCRLattributed difficulties to improve working conditions [42•] which includes modifying physical work conditions [47] to accommodate the physical needs of individuals working with physical limitations secondary to BCRL [48•].

Decision-making and Patient Education Armer et al. [49] offer a review of best practice guidelines for decision-making in assessment and patient education in risk reduction, management, and surveillance for BCRL. Several studies report patient education needs among those with BCRL [50-53]. African American breast cancer survivors $(N=15)$ report perceptions of lacking knowledge about BCRL [50]. Breast cancer survivors receiving BCRL education $(N=35)$ express a preference for highly detailed information [51]. Another report identified that breast cancer survivors $(N=15)$ view BCRL education at the time of lymphedema development to be ill-timed and prefer to receive information about BCRL precautions at the time of breast cancer diagnosis [52]. 
Researchers also report that breast cancer survivors $(N=$ 1065) have low confidence in primary care physicians' ability to address psychosocial aspects of care (i.e., counseling about fears of breast cancer recurrence, sexual concerns, and body image concerns) and suggest a need for educational improvement among providers [53]. Among primary care physicians surveyed $(N=587)$, nearly one-fourth reported low confidence in their ability to manage BCRL [53].

Because BCRL symptoms affect physical and mental wellbeing [54], education can be a psychosocial intervention for breast cancer survivors by reducing anxiety, improving perceptions of controlling lymphedema symptoms, and improving QOL $[55,56]$. Private teaching sessions are the preferred delivery method; however, the preferred timing for receiving the information is variable [51]. Researchers report that breast cancer survivors receiving a Web-based Multimedia Intervention (WBMI) $(n=80)$ perceived higher quality selfcare information than breast cancer survivors receiving pamphlet education $(n=80)$. Furthermore, improved biobehavioral symptoms were observed among WBMI participants compared with control group participants [57•].

\section{BCRL Intervention Research}

Although there is no definitive cure for BCRL, the best BCRL management practice is complete decongestive therapy (CDT) [58]. CDT includes manual lymphatic drainage (MLD), bandaging, compression garments, exercise, and self-care [49]. In the following section, we discuss interventions to manage BCRL, including complementary and integrative health $(\mathrm{CIH})$ approaches, exercise, and surgery.

Complete Decongestive Therapy Seven studies and five RCTs have evaluated CDT and its psychosocial impact [59-67, 68•, $69,70 \bullet$. CDT delivered by a trained physical therapist five times a week for 4 weeks demonstrated a significant reduction in depression, with a positive correlation between arm circumference and depression in a single group trial of 58 women [59]. After 2 weeks of CDT, Park [60] found that the global health domain of QOL improved in women with metastatic disease $(N=59)$. In two single-group CDT studies using different QOL measures, BCRL was reduced with improved general health and functional scores on the EORTC QLQC30 ( $N=60)$ [61] and improved physical functioning, role physical, role emotional, and bodily pain scores on the SF$36(N=40)$ [62]. In a two-group, non-randomized trial $(N=$ 60 ) comparing CDT with conventional therapy, both groups showed improved QOL with the greatest improvement in global, functional, and symptom domains (EORTC QLQ C30, EORTC QLQ-BR23) in the first 4 weeks of the 6week treatment $(p<.05)$ [63].

Stellate Ganglion Block was compared with CDT in an RCT $(N=38)$, and while BCRL improved, there was no improvement in QOL for both groups [64]. An RCT evaluating CDT and active resistance exercise $(N=40)$ found that BCRL was significantly reduced, and QOL (SF-36 physical health and general health) significantly improved, compared with the resistive exercise group [65]. Finally, in a two-group non-randomized trial, participants $(N=31)$ participating in progressive muscle relaxation prior to $\mathrm{CDT}$ had significantly reduced anxiety and depression, compared with those who received only CDT [66]. Other RCTs that evaluated various combinations of the CDT components found improvement in BCRL, but the effect on QOL was mixed. Bahtiyarca [67] demonstrated improved QOL scores in both study groups (compression bandaging vs. compression bandaging with self-lymphatic drainage) $(N=24)$, but there was no significant reduction in anxiety and depression scores. Kinesiotaping, in combination with a pressure garment, resulted in significantly improved overall QOL compared with the group that was only treated with a pressure garment [68 ${ }^{\bullet}$. In a small RCT $(N=45)$ that evaluated arm sleeve compression versus no compression for 1 year, QOL was not improved [69]. However, at 2-year follow-up, the compression group $(n=41)$ had significant improvement of QOL parameters of physical functioning, fatigue, pain, arm, and breast symptoms (EORTC QLQ-C30, QLQBR23) [70•].

Complementary and Integrative Health Approaches Yoga is one of the most frequently studied $\mathrm{CIH}$ approaches used in the management of BCRL; however, the effect of yoga on BCRL and QOL is mixed. Loudon [71] compared the effect of an 8week yoga program $(N=40)$ with a control group, finding that BCRL and QOL (LYMQOL role function and symptom subscales) improved for the yoga group. The yoga group $(N=15)$ also reported improved well-being, increased awareness of their physical body, and improved physical, mental, and social functioning by qualitative interviews [72]. Pasyar [73] conducted a pilot RCT yoga program $(N=40)$ and found that, although BCRL did not improve, role, physical, and emotional functioning did improve. Lastly, Douglass [74] conducted an RCT that evaluated a shorter yoga program of 4 weeks $(N=35)$ and reported no improvement in BCRL; however, women who continued to practice yoga after the 4-week program had better QOL than those who did not.

Other $\mathrm{CIH}$ approaches have been studied. Women $(N=30)$ who received acupuncture in combination with moxibustion in an RCT demonstrated improved BCRL and QOL compared with a control group who received Diosmin $900 \mathrm{mg} 3$ times per day for 30 days [75]. In a randomized placebo-controlled trial $(N=40)$, low laser therapy (LLT) did not reduce BCRL; however, LLT did reduce pain and improved QOL [76•]. In contrast, a separate study comparing LLT vs. MLD vs. MLD and LLT $(N=46)$ found no group differences for psychological and physical symptoms and QOL [77]. 
QOL did not improve among women $(N=107)$ participating in expressive writing about thoughts and feelings (20-min sessions, 4 times per week for 2 weeks) compared with expressive writing about daily activities [78]. Another $\mathrm{CIH}$ approach, reflexology lymphatic drainage (RLD) treatment $(N=$ $26)$, reduced arm swelling and significantly increased wellbeing $(p<.01)[79]$. Finally, an RCT evaluating a selfmanagement BCRL prevention program consisted of training and a BCRL-education booklet (included exercise and massage information) found a decrease in BCRL development and higher QOL in those who participated in the program compared with the control group $(N=61)$ [80].

Physical Activity Various types of exercise have been used in the treatment of BCRL. Reduced BCRL and improved QOL have been reported by exercise studies, including a homebased exercise study $(N=32)$ [81]. Breast cancer survivors $(N=48)$ receiving aqua lymphatic therapy (ALT) reported reduced BCRL and improved emotional and social dimensions of QOL [82]. Pilates $(N=60)$ has also shown to improve QOL and social appearance anxiety more than standard BCRL exercises [83•]. However, some studies showed no improvement in QOL with exercise, including a study comparing physical therapy plus MDT with physical therapy alone $(N=41)$ [84]. Another study reported no group differences in anxiety and depression among breast cancer survivors $(N=$ 45) participating in proprioceptive neuromuscular facilitation exercise with or without light irradiation versus MLD [85]. A 1-year strength training exercise program improved body image and relationship scores compared with the usual care group $(N=295)$; furthermore, the number of arm symptoms was associated with QOL outcomes [86, 87]. Finally, a study by Iacorossi [88 ${ }^{\bullet}$ compared dragon boat racing with exercise $(N=100)$ and reported that women participating in the dragon boat race group had less incidence of BCRL and improved QOL, compared with the exercise group.

Surgery BCRL management with surgery is usually implemented for those women who have extensive BCRL. Our review includes six studies examining the effect of surgery on BCRL, QOL, or psychosocial symptoms. Although all studies found an improvement in BCRL, there were no full-scale RCTs, and sample sizes ranged from 12 to 60 . Liposuction combined with the application of compression garments significantly reduced anxiety and depression, and significantly improved overall wellbeing in 12 women [89]. Hoffner [90•] found that liposuction $(N=60)$ improved QOL (SF-36 general health, bodily pain, vitality, mental health, and social functioning). Lymphaticovenous anastomosis (LVA) was associated with improved QOL in two studies of women with early stage BCRL ( $N=$ $37)$ and $(N=29)$, respectively $[91 \bullet, 92 \cdot]$. In a separate study, 1 year follow-up of women who underwent LVA $(N=20)$ found that QOL remained improved for all domains of the Lymph-ICF questionnaire compared with pre-operative levels [93•]. Researchers studied the effects of vascularized lymph node transplant on QOL in women $(N=50)$ and found improvement $(p<.01)$ in the symptoms domain (LYMQOL) 1 month posttreatment while all other domains (e.g., function, appearance, mood) improved at 3 months $(p<.05)$ and were significant at the 12-month follow-up $(p<.01)[94 \bullet]$.

\section{Discussion}

Our review supports previously identified psychological distress $[6,16-19,20 \cdot 21,22]$ and QOL $[10-15,35-39]$ issues with BCRL [10-15], as noted by Fu et al. [8]. Our review found that beyond previously known psychological and QOL issues, more recent literature examine economic burden, social support, sexuality, and patient education needs. Age is a notable consideration for women with BCRL, as some data suggest that older women (over 60 years) may experience poorer upper extremity function and QOL [9॰] and body image disturbance, compared with younger women [23], while other data suggest that younger women (under 55 years) may experience more pain, fatigue, and psychological distress [20 ]. Importantly, cancer trends in younger women (reported by the North American Association of Central Cancer Registries (NAACCR) as ages 20 to 49) may indicate changing BCRL risk factors associated with age [95]. These findings suggest that further study of symptoms unique to younger and older breast cancer survivors is required as well as the development of age-related interventions to minimize the psychosocial impact of BCRL.

We note recent studies focusing on BCRL economic burden and return-to-work issues [41, 42•, 43-47, 48•], and few studies addressing sexuality concerns [27-29], and body image and self-esteem [23-26]. An interesting finding in this review is the number of reports identifying continued challenges among those with BCRL in receiving education about BCRL and their perceptions about health care providers' abilities to meet their psychosocial care needs [50-56, 57•]. Studies examining social support concerns in women with BCRL report that women continue to experience concerns of lack of privacy, social support, and relationships suggesting the need for social support interventions [30-34]. Reports were mixed regarding associations between the presence of BCRL and QOL, with some studies reporting that self-reported BCRL symptoms are more indicative of QOL scores, while objective measures of BCRL are less predictive of QOL scores [36-40]. Differences in study designs, underpowered samples, and a lack of disease-specific instruments to psychosocial impact of BCRL, including QOL [8], likely account for these findings.

We highlight a study reporting shoulder symptoms associated with poor QOL in those diagnosed with BCRL, which were due to underlying, treatable pathology, such as rotator cuff injury [15]. Although reasons for the initial shoulder 
injuries were not reported [15], other researchers suggest shoulder pathology is related to the duration of BCRL symptoms [96]. These findings have implications for future research examining the post-operative surveillance of the affected upper extremity and early management of BCRL shoulder pain to improve psychosocial outcomes including QOL [96].

CDT has been frequently evaluated to determine its effect on BCRL symptoms and QOL [58, 60-67, 68•, 69, 70•]. Researchers continue to report the safety and acceptability of physical activity among women with BCRL (e.g., aqua lymphatic therapy, Pilates, and dragon boat racing), which has shown to improve BCRL and QOL [82, 83•, 84-87, 88•]. We note an increasing, though a modest number of heterogeneous $\mathrm{CIH}$ interventions (e.g., yoga, low laser therapy) $[71-75,76 \bullet, 77-80]$ and surgical interventions $[89,90 \bullet, 91 \bullet$, 92•, 93•, 94•] to reduce BCRL symptoms and improve QOL. Although findings are promising, the majority of studies are pilot designs and underpowered; thus, more intervention research is needed.

\section{Conclusion}

Recent reports (i.e., within the past several years) focus more on interventions and physical activity to manage psychosocial issues among those with BCRL. Future research is indicated to explore differences in age-related symptoms between younger and older women with BCRL as well as specific, agerelated interventions to reduce the adverse psychosocial experiences of women with BCRL. Likewise, more psychosocialbased intervention research is needed that focuses on sexual concerns, body image, and social support of those with BCRL. Research guiding policies for work-place accommodations for those with physical impairments secondary to lymphedema are also warranted. Although males with BCRL were included in our search criteria, we did not locate eligible articles to include in our review. Future research is recommended to study the psychosocial impact of BCRL among males. Finally, given the recent global pandemicdriven policies for social-distancing and the accompanying uncertainty that surrounds a changing health care system in response to such policies, future psychosocial research among women with BCRL may explore how these new policies affect the psychosocial needs of women with BCRL.

\section{Compliance with Ethical Standards}

Conflict of Interest The authors declare that they have no conflicts of interest.

Human and Animal Rights and Informed Consent This article does not contain any studies with human or animal subjects performed by any of the authors.

\section{References}

Papers of particular interest, published recently, have been highlighted as:

- Of importance

- Of major importance

1. American Cancer Society. Cancer facts \& figures. Atlanta: American Cancer Society; 2020.

2. (NLN). National Lymphedema Network. What is lymphedema?: National Lymphedema Network; 2012 Available from: https:// lymphnet.org/what-is-lymphedema.

3. Casley-Smith JR. Volume alterations in lymphoedema; untreated, and after complex therapy (CPT), benzopyrones, or both. Lymphology. 1994;27:627-31.

4.• Belmonte R, Messaggi-Sartor M, Ferrer M, Pont A, Escalada F. Prospective study of shoulder strength, shoulder range of motion, and lymphedema in breast cancer patients from pre-surgery to 5 years after ALND or SLNB. Supportive Care Canc. 2018;26(9): 3277-87 This study followed 68 SLNB and 44 ALND patients presurgery to 5 years post-surgery and found the ALND group had significant loss of internal rotator strength $(p=.0001)$ and increased limb volume $(p=.03)$ compared with the SLNB group $(p<.001)$. Furthermore, the ALND had more BCRL $(33.3 \%)$ than those receiving SLNB $(3.4 \%)(p<.001)$.

5. Armer JM, Stewart BR. Post-breast cancer lymphedema: incidence increases from 12 to 30 to 60 months. Lymphology. 2010;43(3): 118-27.

6. Bell RJ, Penelope J, Robinson RB, Fradkin P, Schwarz M, Davis SR. Lymphedema: experience of a cohort of women with breast cancer followed for 4 years after diagnosis in Victoria. Australia Supportive Care Canc. 2013;21(7):2017-24.

7. Taghian NR, Miller CL, Jammallo LS, O’Toole J, Skolny MN. Lymphedema following breast cancer treatment and impact on quality of life: a review. Crit Rev Oncol Hematol. 2014;92(3): 227-34.

8. Fu MR, Ridner SH, Hu SH, Stewart BR, Cormier JN, Armer JM. Psychosocial impact of lymphedema: a systematic review of literature from 2004 to 2011. Psycho-Oncology. 2013;22(7):1466-84.

9. Kibar S, Dalyan Aras M, Ünsal DS. The risk factors and prevalence of upper extremity impairments and an analysis of effects of lymphoedema and other impairments on the quality of life of breast cancer patients. Eur J Canc Care. 2017;26(4):1-10 This study of 201 breast cancer survivors demonstrated an incidence rate of $41.3 \%$ and lymphedema was the primary risk factor for developing upper extremity impairment and subsequently diminishing QOL.

10. Velloso FSB, Barra AA, Dias RC. Functional performance of upper limb and quality of life after sentinel lymph node biopsy of breast cancer. Rev Bras Fis. 2011;15(2):146-53.

11. Chachaj A, Małyszczak K, Pyszel K, Lukas J, Tarkowski R, Pudełko M, et al. Physical and psychological impairments of women with upper limb lymphedema following breast cancer treatment. PsychoOnc. 2010;19(3):299-305.

12. Nesvold IL, Fossa SD, Holm I, Naume B, Dahl AA. Arm/shoulder problems in breast cancer survivors are associated with reduced health and poorer physical quality of life. Acta Oncol. 2010;49(3): 347-53.

13. Teo I, Fingeret MC, Liu J, Chang DW. Coping and quality of life of patients following microsurgical treatment for breast cancer-related lymphedema. J Health Psychol. 2016;21(12):2983-93.

14. Penha T, Botter B, Heuts E, Voogd A, von Meyenfeldt M, van der Hulst R. Quality of life in patients with breast cancer-related 
lymphedema and reconstructive breast surgery. J Reconstr Microsurg. 2016;32(6):484-90.

15. Jeong HJ, Sim YJ, Hwang KH, Kim GC. Causes of shoulder pain in women with breast cancer-related lymphedema: a pilot study. Yonsei Med J. 2011;52(4):661-7.

16. Khan F, Amatya B, Pallant JF, Rajapaksa I. Factors associated with long-term functional outcomes and psychological sequelae in women after breast cancer. Breast. 2012;21(3):314-20.

17. Delgado-Sanz MC, García-Mendizábal MJ, Pollán M, Forjaz MJ, López-Abente G, Aragonés N, et al. Heath-related quality of life in Spanish breast cancer patients: a systematic review. Health Qual Life Outcomes. 2011;9(3):1-10.

18. Vassard D, Halgren Olsen M, Zinckernagel L, Vibe-Petersen J, Oksbjerg Dalton S, Johansen C. Psychological consequences of lymphoedema associated with breast cancer: a prospective cohort xtudy. Eur Journal Canc Care. 2010;46(18):3211-8.

19. Thomas A, Job K. Arm function and quality of life among patients after mastectomy. J Clin Diagn Res. 2018;12(4):XC01-XC4.

20. Bjerkeset E, Röhrl K, Schou-Bredal I. Symptom cluster of pain, fatigue, and psychological distress in breast cancer survivors: prevalence and characteristics. Breast Cancer Res Treat. 2020;180(1): 63-71 This study of breast cancer survivors $(\mathbf{n}=\mathbf{8 3 4})$ reported $13 \%$ of participants had symptom clusters of pain, fatigue, and psychological distress. Factors associated with symptoms clusters included BCRL, younger age, working part-time, or being disabled (all $p<.05$ ).

21. Dominick SA, Natarajan L, Pierce JP, Madanat H, Madlensky L. The psychosocial impact of lymphedema-related distress among breast cancer survivors in the WHEL study. Psycho-Oncology. 2014;23(9):1049-56.

22. Taira N, Shimozuma K, Shiroiwa T, Ohsumi S, Kuroi K, Saji S, et al. Associations among baseline variables, treatment-related factors and health-related quality of life 2 years after breast cancer surgery. Breast CancRes Treat. 2011;128(3):735-47.

23. Alcorso J, Sherman KA, Koelmeyer L, Mackie H, Boyages J. Perceived barriers to adherence to breast cancer-related lymphoedema self-management. J Lymphoedema. 2016;11(1): 20-6.

24. Boing L, Pereira GS, Araujo C, Sperandio FF, Loch M, Bergmann A, et al. Factors associated with depression symptoms in women after breast cancer. Rev Saude Publica. 2019;53:30.

25. Anbari AB, Wanchai A, Armer JM. Breast cancer-related lymphedema and quality of life: a qualitative analysis over years of survivorship. Chron Illness. 2019;0(0):1-12.

26. Teo I, Novy DM, Chang DW, Cox MG, Fingeret MC. Examining pain, body image, and depressive symptoms in patients with lymphedema secondary to breast cancer. Psycho-Oncology. 2015;24(11): 1377-83.

27. Yang EJ, Kim SW, Heo CY, Lim JY. Longitudinal changes in sexual problems related to cancer treatment in Korean breast cancer survivors: a prospective cohort study. Support Care Cancer. 2011;19(7):909-18.

28. Radina ME, Fu MR, Horstman L, Kang Y. Breast cancer-related lymphedema and sexual experiences: a mixed-method comparison study. Psycho-Oncology. 2015;24(12):1655-62.

29. Winch CJ, Sherman KA, Koelmeyer LA, Smith KM, Mackie H, Boyages J. Sexual concerns of women diagnosed with breast cancer-related lymphedema. Supportive Care Canc. 2015;23(12): 3481-91.

30. Tsuchiya M, Miyako T. Psychosocial impact of lymphoedema after breast surgery. Curr Breast Cancer Rep. 2016;8(1):47-51.

31. Penha Lopez TR, van Bodegraven J, Winkens B, Heuts EM, Voogd $\mathrm{AC}$, von Meyenfeldt MF. The auality of life in long-term breast cancer survivors with breast cancer related lymphedema. Acta Chir Belg. 2014;114:239-44.
32. Armer JM, Shook RP, Schneider MK, Brooks CW, Peterson J, Stewart BR. Enhancing supportive-educative nursing systems to reduce risk of post-breast cancer lymphedema. SelfCareDepend Care Nurs. 2009;17:6-15.

33. Radina ME, Armer JM, Stewart BR. Making self-care a priority for women at risk of breast cancer-related lymphedema. J Fam Nurs. 2014;20(2):226-49.

34. Tsuchiya M, Horn S, Ingham R. Social disclosure about lymphoedema symptoms: a qualitative study among Japanese breast cancer survivors. Psychol Health Med. 2015;20(6):680-4.

35. Bulley C, Gaal S, Coutts F, Blyth C, Jack W, Chetty U, et al. Comparison of breast cancer-related lymphedema (upper limb swelling) prevalence estimated using objective and subjective criteria and relationship with quality of life. Biomed Res Int. 2013;2013:807569.

36. Sackey H, Johansson H, Sandelin K, Liljegren G, MacLean G, Frisell J, et al. Self-perceived, but not objective lymphoedema is associated with decreased long-term health related quality of life after breast cancer surgery. Eur J Surg Oncol. 2015;41(4):577-84.

37. Popovic-Petrovic S, Kovac A, Kovac N, Tovilovic S, Novakov I, Culibrk D. Secondary lymphedema of the arm, the perception of the disease, self efficacy and depression as determinants of quality of life in patients with breast cancer. Vojnosanit Pregl. 2018;75(10): 961-7.

38. Lee SH, Min YS, Park HY, Jung TD. Health-related quality of life in breast cancer patients with lymphedema who survived more than one year after surgery. J Breast Cancer. 2012;15(4):449-53.

39. Bojinovic-Rodic D, Popovic-Petrovic S, Tomic S, Markez S, Zivanic D. Upper extremity function and quality of life in patients with breast cancer related lymphedema. Vojnosanit Pregl. 2016;73(9):825-30.

40. da Silva RCM, Rezende LF. Assessment of impact of late postoperative physical functional disabilities on quality of life in breast cancer survivors. Tumori. 2014;100:87-90.

41. Fantoni SQ, Peugniez C, Duhamel A, Skrzypczak J, Frimat P, Leroyer A. Factors related to return to work by women with breast cancer in northern France. J Occup Rehabil. 2010;20(1):49-58.

42. Vignes S, Fau-Prudhomot P, Simon L, Sanchez-Brechot ML, Arrault M, Locher F. Impact of breast cancer-related lymphedema on working women. Support Care Cancer. 2020;28(1):79-85 This study of 134 women with BCRL demonstrates that upper limb lymphedema impacts the careers of over half of the women studied $(52.2 \%)$ due to severely impaired arm movement $(p$ $<.01)$.

43. Dean LT, Schmitz KH, Frick KD, Nicholas LH, Zhang Y, Subramanian SV, et al. Consumer credit as a novel marker for economic burden and health after cancer in a diverse population of breast cancer survivors in the USA. J Cancer Surviv. 2018;12(3): 306-15.

44. Azarkish F, Mirzaii Najmabadi K, Latifnejad Roudsari R, Homaei SF. Factors related to return to work in women after breast cancer in Iran. Iran Red Crescent Med J. 2015;17(9):e19978.

45. Peugniez C, Leroyer A, Skrzypczak J, Duprey M, Bonneterre J. Return to work after treatment for breast cancer: single center experience in a cohort of 273 patients. Bull Cancer. 2011;98(7):E69 79 .

46. Schmitz KH, DiSipio T, Gordon LG, Hayes SC. Adverse breast cancer treatment effects: the economic case for making rehabilitative programs standard of care. Support Care Cancer. 2015;23(6): 1807-17.

47. Zomkowski K, Cruz de Souza B, Pinheiro da Silva F, Moreira GM, de Souza Cunha N, Sperandio FF. Physical symptoms and working performance in female breast cancer survivors: a systematic review. Disabil Rehabil. 2018;40(13):1485-93.

48. Sun Y, Shigaki CL, Armer JM. Return to work among breast cancer survivors: a literature review. Support Care Cancer. 2017;25(3): 
709-18 This literature review of 25 articles reports on breast cancer survivors ability to return to work, work ability, and work performance and highlights a need for feasible interventions for supporting survivors's return to work.

49. Armer JM, Hulett JM, Bernas M, Ostby P, Stewart BR, Cormier J. Best practice guidelines in assessment, risk reduction, management, and surveillance for post-breast cancer lymphedema. Curr Breast Canc Res. 2013;5:134-44.

50. Adams N, Gisiger-Camata S, Hardy CM, Thomas TF, Jukkala A, Meneses K. Evaluating survivorship experiences and needs among rural African American breast cancer survivors. J Cancer Educ. 2017;32(2):264-71.

51. White JA, Matthews E, Wallace L, Poormon L. Informational needs of breast cancer patients related to lymphedema. Rehabil Oncol. 2015;33(1):58-63.

52. Ostby PL, Armer JM, Smith K, Stewart BR. Patient perceptions of barriers to self-management of breast cancer-related lymphedema. WestJ Nurs Res. 2018;40(12):1800-17.

53. Smith SL, Murchison S, Singh-Carlson S, Alexander C, Wai ES. Survivorship care in breast cancer. Can Fam Physician. 2015;61: e277-83.

54. Panobianco MS, Campacci N, Fangel LMV, Prado MAS, Almeida AMD, Gozzo TDO. Quality of life of women with lymphedema after surgery for breast cancer. Revista Da Rede de Enfermagem Do Nordeste. 2014;15(2):206-13.

55. Bland KL, Kosir MA. Improving the quality of life in breast cancer survivors at risk for lymphedema. Surgery. 2019;166(4):686-90.

56. Lin CY, Liu HE, Cheng MH. Factors associated with professional healthcare advice seeking in breast cancer-related lymphedema. J SurgOncol. 2019;121:67-74.

57. Ridner SH, Dietrich MS, Davis AJ, Sinclair V. A randomized clinical trial comparing the impact of a web-based multimedia intervention versus an educational pamphlet on patient outcomes in breast cancer survivors with chronic secondary lymphedema. J Women's Health. 2019;0(0):1-11 This intervention study examines the effects of a web-based multimedia intervention for BCRL patients and demonstrated superiority to using pamphlets in terms of providing high-quality self-care information $(p=.001)$.

58. Chang CJ, Cormier JN. Lymphedema interventions: exercise, surgery, and compression devices. Semin Oncol Nurs. 2013;29(1):2840.

59. Atalay OT, Özkir A, Başakçi cAlik B, Baskan E, Taşkin H. Effects of phase I complex decongestive physiotherapy on physical functions and depression levels in breast cancer related lymph edema. J PhysTher Sci. 2015;27(3):865-70.

60. Park JE, Jang HJ, Seo KS. Quality of life, upper extremity function and the effect of lymphedema treatment in breast cancer related lymphedema patients. Ann Rehabil Med. 2012;36:240-7.

61. Yesil H, Eyigor S, Caramat I, Isik R. Effects of complex decongestive therapy on quality of life, depression, neuropathic pain, and fatigue in women with breast cancer-related lymphedema. Turk $\mathbf{J}$ Phys Med Rehabil. 2017;63(4):329-34.

62. Tunay VB, Akbayrak T, Kaya S. The effect of multidimensional physiotherapy program on shoulder function, pain, and lymphedema after surgery in elderly breast cancer patients. Top GeriatrRehabil. 2012;28(4):281-6.

63. Melam GR, Buragadda S, Alhusaini AA, Arora N. Effect of complete decongestive therapy and home program on health- related quality of life in post mastectomy lymphedema patients. BMC Womens Health. 2016;16:23.

64. Park MW, Shi-Uk L, Sohyun K, Kwan SS. Comparison between the effectiveness of complex decongestive therapy and stellate ganglion block in patients with breast cancer-related lymphedema: a randomized controlled study. Pain Physician. 2019;22:255-63.
65. Kim DS, Sim YJ, Jeong HJ, Kim GC. Effect of active resistive exercise on breast cancer-related lymphedema: a randomized controlled trial. Arch Phys Med Rehabil. 2010;91(12):1844-8.

66. Abbasi B, Mirzakhany N, Oshnari LA, Irani A, Hosseinzadeh S, Tabatabaei SM, et al. The effect of relaxation techniques on edema, anxiety and depression in post-mastectomy lymphedema patients undergoing comprehensive decongestive therapy: a clinical trial. PLoS One. 2018;13(1):e0190231.

67. Bahtiyarca ZT, Can A, Ekșioğlu E, Çakcı A. The addition of selflymphatic drainage to compression therapy instead of manual lymphatic drainage in the first phase of complex decongestive therapy for treatment of breast cancer-related lymphedema: a randomized controlled, prospective study. Turkish J Phys Med Rehab. 2019;65(4):309-17.

68. Tantawy SA, Abdelbasset WK, Nambi G, Kamel DM. Comparative study between the effects of kinesio taping and pressure garment on secondary upper extremity lymphedema and quality of life following mastectomy: a randomized controlled trial. Integr Canc Ther. 2019;18:1-10 This RCT ( $\boldsymbol{n}=\mathbf{6 6})$ demonstrated Kinesiotaping reduced limb circumference, improved QOL, and improved hand grip strength among women with BCRL $(p<.05)$ compared with pressure garments.

69. Ochalek K, Gradalski T, Szygula Z, Partsch H. Physical activity with and without arm sleeves: compliance and quality of life after breast cancer surgery - a randomized controlled trial. Lymphat Res Biol. 2018;16(3):294-9.

70. Ochalek K, Partsch H, Gradalski T, Szygula Z. Do compression sleeves reduce the incidence of arm lymphedema and improve quality of life? Two-year results from a prospective randomized trial in breast cancer survivors. LymphatRes Bio. 2019;17(1):70 7 This pilot RCT $(n=41)$ offers preliminary data in support that wearing light compression sleeves reduces the incidence of early post-operative lymphedema and improves QOL.

71. Loudon A, Barnett T, Piller N, Immink MA, Visentin D, Williams AD. The effect of yoga on women with secondary arm lymphoedema from breast cancer treatment. BMC Compl Alternative Med. 2012;12(1):1-9.

72. Loudon A, Barnett T, Williams A. Yoga, breast cancer-related lymphoedema and well-being: a descriptive report of women's participation in a clinical trial. J Clin Nurs. 2017;26(23):4685-95.

73. Pasyar N, Tashnizi NB, Mansouri P, Tahmasebi S. Effect of yoga exercise on the quality of life and upper extremity volume among women with breast cancer related lymphedema: a pilot study. Eur J Oncol Nurs. 2019;42:103-9.

74. Douglass J, Immink M, Piller N, Ullah S. Yoga for women with breast cancer related lymphoedema: a preliminary 6-month study. J Lymphoedema. 2012;7(2):30-8.

75. Yao $\mathrm{C}, \mathrm{Xu} \mathrm{Y}$, Chen L, Jiang H, Ki CS, Byun JS, et al. Effects of warm acupuncture on breast cancer-related chronic lymphedema: a randomized controlled trial. Curr Oncol. 2016;23(1):e27-34.

76. Storz MA, Gronwald B, Gottschling S, Schöpe J, Mavrova R, Baum S. Photobiomodulation therapy in breast cancer-related lymphedema: a randomized placebo-controlled trial. Photodermatol Photoimmunol Photomed. 2017;33(1):32-40 This study is relatively unique in examining effects of photobiomodulation therapy among 40 women with lymphedema. Although null findings were reported for limb volume in this pilot RCT, PBMT was associated with improved QOL and reduced pain.

77. Ridner SH, Poage-Hooper E, Kanar C, Doersam JK, Bond SM, Dietrich MS. A pilot randomized trial evaluating low-level laser therapy as an alternative treatment to manual lymphatic drainage for breast cancer-related lymphedema. Oncol Nurs Forum. 2013;40(4):383-93. 
78. Sohl SJ, Dietrich MS, Wallston KA, Ridner SH. A randomized controlled trial of expressive writing in breast cancer survivors with lymphedema. Psychol Health. 2017;32(7):826-42.

79. Whatley J, Street R, Kay S, Harris PE. Use of reflexology in managing secondary lymphoedema for patients affected by treatments for breast cancer: a feasibility study. Compl Ther Clin Pract. 2016;23:1-8.

80. Temur K, Kapucu S. The effectiveness of lymphedema selfmanagement in the prevention of breast cancer-related lymphedema and quality of life: a randomized controlled trial. Eur J Oncol Nurs. 2019;40:22-35.

81. Gautam AP, Maiya AG, Vidyasagar MS. Effect of home-based exercise program on lymphedema and quality of life in female postmastectomy patients: pre-post intervention study. J Rehabil Res Dev. 2011;48(10):1261-8.

82. Tidhar D, Katz-Leurer M. Aqua lymphatic therapy in women who suffer from breast cancer treatment-related lymphedema: a randomized controlled study. Support Care Cancer. 2010;18(3):383-92.

83. Sener HO, Malkoc M, Ergin G, Karadibak D, Yavuzsen T. Effects of clinical pilates exercises on patients developing lymphedema after breast cancer treatment: a randomized clinical trial. J Breast Health. 2017;13(1):16-22 This small RCT of 60 women with BCRL demonstrated that Pilates is safe for those with BCRL, improves social appearance anxiey and QOL, and offers preliminary data that Pilates may more effective in BCRL symptoms than standard lymphedema exercises $(p<.05)$.

84. Cho Y, Do J, Jung S, Kwon O, Jeon JY. Effects of a physical therapy program combined with manual lymphatic drainage on shoulder function, quality of life, lymphedema incidence, and pain in breast cancer patients with axillary web syndrome following axillary dissection. Support Care Cancer. 2016;24(5):2047-57.

85. Ha K, Choi S. The effect of a PNF technique program after mastectomy on lymphedema patients' depression and anxiety. J PhysTher Sci. 2014;26(7):1065-7.

86. Hormes JM, Bryan C, Lytle LA, Gross CR, Ahmed RL, Troxel AB, et al. Impact of lymphedema and arm symptoms on quality of life in breast cancer survivors. Lymphology. 2010;43:1-13.

87. Speck RM, Gross CR, Hormes JM, Ahmed RL, Lytle LA, Hwang WT, et al. Changes in the body image and relationship scale following a one-year strength training trial for breast cancer survivors with or at risk for lymphedema. Breast Canc ResTreat. 2010;121(2):421-30.

88. Iacorossi L, Gambalunga F, Molinaro S, Domenico RD, Giannarelli D, Fabi A. The effectiveness of the sport 'dragon boat racing' in reducing the risk of lymphedema incidence: an observational study. Cancer Nurs. 2019;42(4):323-31 This novel complementary approach demonstrates the dragon boat racing is safe among women with BCRL $(n=100)$, and dragon boat racing is associated with a decreased incidence of lymphedema and improved QOL among women participating in dragon boat racing compared with women who did not participate in dragon boat racing ( $4 \%$ vs. $26 \%$, respectively).
89. Schaverien MV, Munro KJ, Baker PA, Munnoch DA. Liposuction for chronic lymphoedema of the upper limb: 5 years of experience. J Plast Reconstr Aesthet Surg. 2012;65(7):935-4.

90. Hoffner M, Bagheri S, Hansson E, Manjer J, Troëng T, Brorson H. SF-36 shows increased quality of life following complete reduction of postmastectomy lymphedema with liposuction. Lymphat Res Biol. 2017;15(1):87-98 This study of $\mathbf{6 0}$ women with BCRL demonstrates that liposuction and controlled compression therapy $(\mathrm{CCT})$ reduce lymphedema and improve QOL $(p<.05)$.

91. Phillips GSA, Gore S, Ramsden A, Furniss D. Lymphaticovenular anastomosis improves quality of life and limb volume in patients with secondary lymphedema after breast cancer treatment. Breast $\mathrm{J}$. 2019;25(5):859-64 This study offers preliminary data supporting the use of lymphaticovenular anastomosis (LVA) to reduce limb volume among 37 women with early stage lymphedema $(p<.005)$ and was associated with improved QOL $(p<.005)$.

92. Winters H, Tielemans HJP, Hameeteman M, Paulus VAA, Beurskens CH, Slater NJ, et al. The efficacy of lymphaticovenular anastomosis in breast cancer-related lymphedema. Breast Cancer Res Treat. 2017;165(2):321-7 This pilot study of 29 women with unilateral lymphedema demonstrated that $\mathrm{LVA}$ reduces limb volume ( $p=.00$ at 6 months and $p=.02$ at 12 months) among women with BCRL and improves QOL.

93. Cornelissen AJM, Kool M, Penha TRL, Keuter XHA, Piatkowski AA, Heuts E, et al. Lymphatico-Venous anastomosis as treatment for breast cancer-related lymphedema: a prospective study on quality of life. Breast Cancer Res Treat. 2017;163(2) This pilot study of LVA in 20 women with early stage BCRL did not demonstrate a significant decrease in limb volume. However, $85 \%$ of participants were able to discontinue the use of compression stockings and were associated with improved QOL.

94. Gratzon A, Schultz J, Secrest K, Lee K, Feiner J, Klein RD. Clinical and psychosocial outcomes of vascularized lymph node transfer for the treatment of upper extremity lymphedema after breast cancer therapy. Ann Surg Oncol. 2017;24(6):1475-81 This study examined vascularized lymph node transfer (VLNT) to the axilla in 50 women with BCRL and demonstrated decreased arm volume by $58.7 \%$ at 12 months. Corresponding decreases in pain $(p<.01)$ and heaviness $(p<.01)$ were observed. QOL improved over time $(p<.01)$. Minor wound complications occurred in 17 patients.

95. North American Association of Central Cancer Registries (NAACCR). Annual report to the nation on the status of cancer. Springfield: NAACCR; 2018.

96. Jang DH, Kim MW, Oh SJ, Kim JM. The influence of arm swelling duration on shoulder pathology in breast cancer patients with lymphedema. PLoS One. 2015;10(11):e0142950-e.

Publisher's Note Springer Nature remains neutral with regard to jurisdictional claims in published maps and institutional affiliations. 